\title{
Nanocompósitos de Blendas HDPE/LLDPE e OMMT - Parte II: Avaliação das Propriedades Térmica, Óticas e de Transporte a Gases
}

\author{
Fabio R. Passador, Daniel R. Travain, Eduardo H. Backes, Adhemar C. Ruvolo Filho, Luiz A. Pessan \\ Departamento de Engenharia de Materiais, UFSCar
}

\begin{abstract}
Resumo: Nanocompósitos de blendas de polietileno de alta densidade (HDPE) com polietileno linear de baixa densidade (LLDPE) e OMMT (montmorilonita organofílica) foram preparados sob fusão em extrusora de duplarosca, utilizando HDPE-g-MA como agente compatibilizante. Os nanocompósitos foram caracterizados através das propriedades térmicas, óticas e de transporte de gases. A blenda HDPE/LLDPE e os nanocompósitos das blendas HDPE/LLDPE como esperado comportam-se como barreira ao vapor de água e são permeáveis ao $\mathrm{CO}_{2}$ e $\mathrm{O}_{2}$. A adição de nanoargila modificou o grau de cristalinidade da matriz polimérica dos nanocompósitos e a diminuição do coeficiente de permeabilidade foi atribuída ao aumento do grau de cristalinidade e do aumento ao caminho difusional para as moléculas do gás passarem pelo filme polimérico. O caminho difusional mais longo devido ao aumento da tortuosidade está relacionado a uma boa dispersão da carga inorgânica, boa molhabilidade desta pela matriz e forte interações na interface. Modelos teóricos de permeabilidade propostos por Nielsen e Bharadwaj foram utilizados para estimar a razão de aspecto da nanoargila nos nanocompósitos e forneceram resultados que se correlacionam bem com as morfologias observadas por microscopia eletrônica de transmissão.
\end{abstract}

Palavras-chave: Nanocompósitos, HDPE/LLDPE, propriedades de transporte, Modelo de Nielsen, Modelo de Bharadwaj.

\section{HDPE/LLDPE Blend-based Nanocomposites - Part II: Evaluation of Thermal, Optical and Transport Properties}

\begin{abstract}
High density polyethylene/ linear low density polyethylene (HDPE/LLDPE) blend-based nanocomposites were prepared in a twin-screw, using HDPE-g-MA as compatibilizer agent. The nanocomposites were characterized through thermal, optical and transport properties. The HDPE/LLDPE blend and the nanocomposites behave as a barrier to water vapor but are permeable to $\mathrm{CO}_{2}$ e $\mathrm{O}_{2}$. The addition of organoclay modified the crystallinity degree of the polymer matrix of the nanocomposites. The reduction of the permeability coefficient of the nanocomposites was correlated to the increase in the crystallinity of the polymer matrix and to the increase in diffusional path of the gas due to the increase in the tortuosity related to a good dispersion of the clay platelets in the matrix. These effects are related also to a good wettability of the filler by the polymeric matrix and strong interactions at the interface. Theorical permeability models proposed by Nielsen and Bharadwaj were used to estimate the aspect ratio of the organoclay in the nanocomposites with correlate well with the images observed through transmission electron microscopy.
\end{abstract}

Keywords: Nanocomposites, HDPE/LLDPE, transport properties, Nielsen model, Bharadwaj model.

\section{Introdução}

Embalagens flexíveis de poliolefinas são largamente utilizadas na indústria de alimentos, pois apresentam baixa permeabilidade intrínseca a vapor de água devido à natureza hidrofóbica das poliolefinas ${ }^{[1-4]}$. A formação de um nanocompósito polimérico com estrutura intercalada e/ou esfoliada com argila bem dispersa na matriz polimérica usualmente promove modificações significativas em suas propriedades térmicas, mecânicas e de barreira, devido a possibilidade de gerar elevada área de interação interfacial e a formação de interações adequadas polímero-argila nestes sistemas ${ }^{[1-7]}$. Entretanto a obtenção de nanocompósitos poliolefínicos esfoliados representa normalmente um grande desafio, pois por serem hidrofóbicas, as interações entre a matriz poliolefínica e a superfície polar dos silicatos são fracas, e assim, a simples aplicação de cisalhamento na mistura no estado fundido dificilmente levaria à delaminação das lamelas de argila, sendo necessária a adição de agentes compatibilizantes para melhorar as interações entre a matriz polimérica e a nanocarga ${ }^{[8-10]}$.

As propriedades de transporte de moléculas de gases e vapores em filmes e membranas poliméricas são dependentes fortemente da estrutura molecular da matriz polimérica sendo também muito sensíveis às suas alterações ${ }^{[11]}$. A adição de argila em sistemas poliméricos, na grande maioria dos casos, leva a alterações de suas propriedades de transporte. A permeabilidade de pequenas moléculas em nanocompósitos com argila pode sofrer reduções substanciais em relação ao polímero puro devido à grande razão de aspecto e o caráter

Autor para correspondência: Fabio R. Passador, Departamento de Engenharia de Materiais, Universidade Federal de São Carlos - UFSCar, Rod. Washington Luis, Km 235, CP 676, CEP 13565-905, São Carlos, SP, Brasil, e-mail: fabiopassador@gmail.com 
impermeável das lamelas de argila, que são responsáveis pelos efeitos de tortuosidade quando estão dispersas numa matriz polimérica ${ }^{[12-14]}$. Diferentes modelos teóricos têm sido propostos na literatura para expressar o fator de tortuosidade como uma função da forma, orientação, estado de dispersão e fração volumétrica das partículas impermeáveis.

Nielsen ${ }^{[15]}$ desenvolveu um modelo de permeabilidade de compósitos considerando o efeito da tortuosidade, sendo utilizado para as medidas em nanocompósitos através da expressão:

$$
\frac{P}{P_{0}}=\frac{1-\phi_{N C}}{\tau}=\frac{\left(1-\phi_{N C}\right)}{\left(1+\frac{L}{2 W} \phi_{N C}\right)}
$$

onde $P$ e $P_{0}$ são as permeabilidades do nanocompósito e do polímero puro, respectivamente. $O$ fator de tortuosidade $\left(\tau=\frac{d^{\prime}}{d}=1+\frac{L}{2 W} \phi_{N C}\right)$ é definido como a razão da distância ou caminho real ( $d^{\prime}$ ) que o penetrante deve atravessar para uma distância mais curta $(d)$ que este atravessaria na ausência do silicato em camadas e é expresso em termos do comprimento $(L)$, espessura das camadas de argila $(W)$ e da fração volumétrica da carga $\left(\Phi_{N C}\right)$. Cargas com elevado fator de forma $(L / W)$ representam um elevado fator de tortuosidade e, consequentemente, a permeabilidade do nanocompósito será menor que a do polímero puro. A Figura 1 ilustra o modelo do caminho difusional de um penetrante através do nanocompósito.

O modelo proposto por Nielsen apresenta algumas limitações, uma vez que as camadas de argila se encontram perfeitamente orientadas na direção transversal à direção da permeação, apresentam mesmo tamanho, a difusividade da matriz não muda pela presença da argila e que não ocorre transporte preferencial nas interfaces polímero/argila.

Bharadwaj ${ }^{[16]}$ desenvolveu um modelo para predizer a permeabilidade baseado estritamente na tortuosidade. A redução da permeabilidade é decorrência de um longo caminho de difusão que o penetrante deve percorrer na presença de carga. A morfologia apresentada pelas lamelas de argila é particularmente eficiente para maximizar o comprimento do caminho devido a grande razão entre o comprimento e a largura comparada com outras cargas como esferas e cubos. O modelo assume que as lamelas estão localizadas preferencialmente em uma orientação e coincidem com a direção da difusão. Isso resulta numa maior tortuosidade. Qualquer desvio do arranjo onde as lamelas estejam localizadas poderia deteriorar as propriedades de barreira, sendo necessária a adição de um parâmetro (S) que está relacionado com as possíveis variações na orientação, $S=\frac{1}{2}\left\langle 3 \cos ^{2} \theta-1\right\rangle$, onde $\theta$ representa o ângulo entre os vetores unitários da orientação preferencial (n) e a normal ás lamelas (p), como mostrado na Figura 2.

Essa função pode assumir o valor $1(\theta=0)$, indicando perfeita orientação das unidades de vetor normal das lamelas $\mathbf{p}$ com $\mathbf{n}$, pode assumir o valor $-1 / 2(\theta=\pi / 2)$, indicando orientação perpendicular ou ortogonal, e

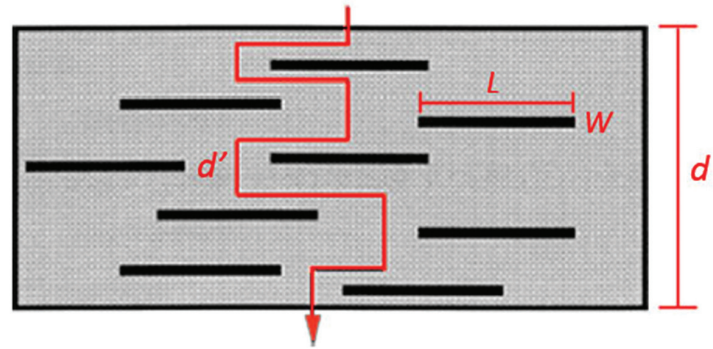

Figura 1. Modelo do caminho difusional de um penetrante através do nanocompósito ${ }^{[15]}$.
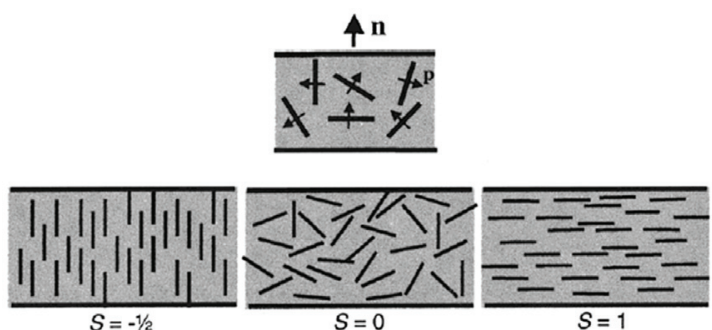

Figura 2. Definição da direção da orientação preferencial das lamelas de silicato ${ }^{[16]}$.

assumir o valor 0 (zero) que indica uma orientação randômica das lamelas. O fator de tortuosidade é modificado para incluir a ordem de orientação, e a permeabilidade relativa é dada por:

$$
\frac{P}{P_{0}}=\frac{1-\phi_{N C}}{1+\frac{L}{2 W} \phi_{N C}\left(\frac{2}{3}\right)\left(S+\frac{1}{2}\right)}
$$

A expressão acima se reduz a equação 1 quando $S=1$ (arranjo planar) e converge para aproximadamente a permeabilidade do polímero puro, quando $S=-1 / 2$ (arranjo ortogonal).

O objetivo principal deste trabalho foi avaliar o efeito da adição do agente compatibilizante HDPE-g-MA e do teor de argilomineral nas propriedades de transporte de pequenas moléculas em nanocompósitos de blendas HDPE/LLDPE e utilizar os modelos de Nielsen e Bharadwaj para estimar a razão de aspecto dos tactóides e/ou lamelas da nanoargila nesses nanocompósitos.

\section{Experimental}

\section{Materiais}

Para a preparação dos nanocompósitos de blenda HDPE/LLDPE e OMMT foram utilizados:

- Polietileno de alta densidade (HDPE) com índice de fluidez de $0,38 \mathrm{~g} / 10 \mathrm{~min}\left(190^{\circ} \mathrm{C} / 2,16 \mathrm{~kg}\right)$, de especificação DMDA 6200 NT-7, fornecido pela Dow Chemical;

- Polietileno linear de baixa densidade (LLDPE). Copolímero de buteno-1, produzido pelo processo de solução com índice de fluidez de 29 g/10min $\left(190^{\circ} \mathrm{C} / 2,16 \mathrm{~kg}\right)$, de especificação IC-32, fornecido pela Braskem; 
- Polietileno de alta densidade enxertado com anidrido maleico (HDPE-g-MA) utilizado como agente compatibilizante, com MFI = 3-6 g/10min $\left(190^{\circ} \mathrm{C} / 2,16 \mathrm{~kg}\right)$, de especificação Polybond ${ }^{\circledR} 3009$, funcionalizado com $1 \%$ em massa de anidrido maleico, da Crompton Corporation;

- Argila montmorilonita natural modificada com um sal quaternário de amônio (OMMT), de nome comercial Cloisite ${ }^{\circledR}$ 20A produzida pela Southern Clay Products Inc., com densidade aparente de aproximadamente $0,32 \mathrm{~g} / \mathrm{cm}^{3}$, com perda de massa durante a queima de $38 \%$ e com espaçamento basal de $2,32 \mathrm{~nm}$.

\section{Preparação dos nanocompósitos}

A blenda HDPE/LLDPE e os nanocompósitos foram preparados através da mistura no estado fundido em uma extrusora de dupla rosca co-rotacional MT19TC da marca B\&P Process Equipament and Systems com roscas com diâmetro de $19 \mathrm{~mm}$ e $\mathrm{L} / \mathrm{D}=25$. Foram utilizados os seguintes parâmetro de processo: velocidade das roscas de $120 \mathrm{rpm}$, vazão de $0,7 \mathrm{~kg} / \mathrm{h}$ e perfil de temperatura de $180^{\circ} \mathrm{C} / 190^{\circ} \mathrm{C} / 190^{\circ} \mathrm{C} / 200^{\circ} \mathrm{C} / 210^{\circ} \mathrm{C}$.

Os nanocompósitos com a blenda HDPE/LLDPE e OMMT utilizando o HDPE-g-MA como agente compatibilizante foram preparados em duas etapas. $\mathrm{Na}$ primeira etapa de mistura foi preparado um compósito de HDPE/HDPE-g-MA/OMMT e em uma segunda etapa de mistura foi adicionado o LLDPE ao sistema preparado na primeira etapa. A razão mássica entre HDPE/LLDPE utilizada foi de 3:1 e a razão mássica entre OMMT/HDPE-g-MA foi de 1:2. Foram utilizados três teores de OMMT: 2,5; 5,0 e 7,5 \% em massa que foram designados por $\mathrm{NC}(2,5), \mathrm{NC}(5,0)$ e $\mathrm{NC}(7,5)$, respectivamente. Nanocompósitos de blenda HDPE/ LLDPE e OMMT sem adição de agente compatibilizante foram preparados em uma única etapa, na razão mássica HDPE/LLDPE de 3:1 e três teores de OMMT: 2,5; 5,0 e $7,5 \%$ em massa que foram designados como $\operatorname{NSC}(2,5)$, $\operatorname{NSC}(5,0)$ e $\operatorname{NSC}(7,5)$, respectivamente. Os materiais poliméricos iniciais para as preparações das misturas e a blenda HDPE/LLDPE (75/25\%) também foram preparados nas mesmas condições para serem utilizadas como referência no estudo. A caracterização estrutural dos nanocompósitos foi realizada e relatada em trabalho anterior em Passador et al. ${ }^{[17]}$.

\section{Extrusão de filmes planos}

Filmes planos uniaxiais de HDPE, LLDPE, da blenda HDPE/LLDPE e dos nanocompósitos foram produzidos em uma extrusora de rosca única da AX Plásticos, modelo LAB-16, acoplada a uma matriz plana, possuindo 3 zonas de aquecimento com temperatura variando de 235 a $240^{\circ} \mathrm{C}$, rotação de $60 \mathrm{rpm}$ e velocidade de estiramento de $2,5 \mathrm{rpm}$.

\section{Propriedades térmicas}

As propriedades térmicas dos polímeros iniciais, da blenda entre eles e dos nanocompósitos foram avaliadas por ensaios de análise termogravimétrica (TGA) e calorimetria exploratória diferencial (DSC).
A estabilidade térmica dos nanocompósitos e o teor de argila organofílica incorporada foram verificados através de análise termogravimétrica. $\mathrm{O}$ ensaio foi realizado em um equipamento da TA Instruments, modelo TGA Q50, a uma taxa de aquecimento de $20^{\circ} \mathrm{C} / \mathrm{min}$, a partir da temperatura ambiente até $600^{\circ} \mathrm{C}$, sob atmosfera de $\mathrm{N}_{2}$.

Para as análises de DSC, utilizou-se o equipamento da TA Instruments, modelo QS100 com nitrogênio como gás de arraste, em fluxo contínuo de $50 \mathrm{ml} . \mathrm{min}^{-1}$. As amostras foram inicialmente aquecidas até $200^{\circ} \mathrm{C}$ a uma taxa de aquecimento de $10^{\circ} \mathrm{C} / \mathrm{min}$, e mantidas nesta temperatura por 5 minutos. A seguir as amostras foram resfriadas até $40^{\circ} \mathrm{C}$ a uma taxa de $10^{\circ} \mathrm{C} / \mathrm{min}$ para determinação da temperatura de cristalização $\left(\mathrm{T}_{\mathrm{c}}\right)$ e novamente foram aquecidas até $200^{\circ} \mathrm{C}$ a uma taxa de $10^{\circ} \mathrm{C} / \mathrm{min}$. $\mathrm{O}$ valor da temperatura de fusão cristalina $\left(\mathrm{T}_{\mathrm{m}}\right)$ foi obtido no segundo ciclo de aquecimento.

\section{Propriedades de transporte}

Os polímeros iniciais, a blenda entre eles e os nanocompósitos preparados foram caracterizados quanto as suas propriedades de permeabilidade ao $\mathrm{O}_{2}$, ao $\mathrm{CO}_{2} \mathrm{e}$ ao vapor de água.

Permeação de Vapor de Água : As medidas de permeação ao vapor de água foram realizadas de acordo com a norma ASTM E 96/E 96M-05 $5^{[18]}$. Amostras dos filmes foram cortadas e acopladas a copos de vidro apropriados para este teste com auxílio de resina epóxi, sendo que após a secagem da resina epóxi, os recipientes foram pesados e deixados em um dessecador, com umidade relativa controlada em $17 \%$. O acompanhamento gravimétrico da variação de massa do sistema foi feito utilizando-se uma balança eletrônica. As medidas foram feitas a cada 24 horas por um período de 10 dias. A temperatura foi controlada pela climatização da sala, com um valor médio de $23 \pm 1^{\circ} \mathrm{C}$.

Permeação de Oxigênio: As medidas de permeação de oxigênio foram feitas utilizando um equipamento OX-TRAN ${ }^{\circledR}$, modelo $2 / 21 \mathrm{~T}$ da MOCON $^{\circledR}$. As taxas de permeabilidade ao oxigênio foram determinadas por método coulométrico, segundo procedimento descrito na norma ASTM D 3985-05 ${ }^{[19]}$. As amostras dos filmes foram condicionadas por 24 horas a $23^{\circ} \mathrm{C}$ em ambiente desumidificado. Os corpos de prova foram montados e colados em máscaras de alumínio com área efetiva de permeação de $5 \mathrm{~cm}^{2}$. O fluxo de oxigênio durante o ensaio foi de $10 \mathrm{~cm}^{3} / \mathrm{min}$, utilizando o modo de convergência por ciclos.

Permeação de Dióxido de Carbono: As medidas de permeação de dióxido de carbono foram feitas utilizando um equipamento PERMATRAN-C ${ }^{\circledR}$, modelo 4/41 da $\mathrm{MOCON}^{\circledR}$. Neste ensaio o $\mathrm{CO}_{2}$ que passa através do filme é conduzido para o sensor infravermelho por um fluxo de nitrogênio ultra seco, segundo procedimento descrito na norma ASTM F 2476-05 ${ }^{[20]}$. As amostras dos filmes foram condicionadas por 24 horas a $23^{\circ} \mathrm{C}$ em ambiente desumidificado antes dos testes. Os corpos de prova foram montados e colados em máscaras de alumínio com área efetiva de permeação de $5 \mathrm{~cm}^{2}$. O fluxo de dióxido de carbono durante o ensaio foi de $50 \mathrm{~cm}^{3} / \mathrm{min}$, utilizando o modo de convergência por ciclos. 


\section{Propriedades óticas}

A influência da adição de argilomineral nas propriedades óticas dos filmes obtidos foi medida de acordo com a norma ASTM D 1003-07[21], em um opacímetro da marca KYK-Gardner, modelo Haze Gard-plus. Foram obtidos valores da transmitância, da opacidade e da claridade das amostras.

\section{Resultados e Discussão}

\section{Avaliação das propriedades térmicas}

Através da análise termogravimétrica foi possível obter informações sobre a temperatura de início de decomposição térmica irreversível e do teor de resíduo inorgânico das composições estudadas. Esses dados foram utilizados para o cálculo do grau de cristalinidade dos nanocompósitos e principalmente nos modelos das propriedades de transporte.

A Figura 3 apresenta as curvas termogravimétricas dos materiais estudados.

Os valores da temperatura de início de decomposição térmica irreversível e do teor de resíduo são apresentados na Tabela 1.

A temperatura de início de decomposição da blenda HDPE/LLDPE apresentou valores intermediários aos materiais individuais utilizados. Para as composições compatibilizadas observou-se que quanto maior o teor de argilomineral empregado, menor a temperatura de início de degradação do material. Pode-se notar que a adição do agente compatibilizante modificou a morfologia das composições, e auxiliou no aumento da temperatura de início de decomposição térmica, em comparação aos nanocompósitos sem a adição de agentes compatibilizantes. Para as composições compatibilizadas observou-se que quanto maior o teor de argilomineral empregado, menor a temperatura de início de decomposição do material, sendo que tal fato está relacionado com a relação agente compatibilizante/ nanoargila utilizada que seguiu a proporção de 2:1. Quanto maior foi o teor de nanoargila utilizada, também maior o teor de agente compatibilizante no sistema e, por consequência, maior o teor de anidridro maleico o qual é decomposto em temperaturas inferiores ao material polimérico original.

Tabela 1. Temperatura de início de decomposição térmica e teor de resíduo dos materiais iniciais e dos nanocompósitos estudados.

\begin{tabular}{lcc}
\hline \multicolumn{1}{c}{ Amostra } & $\mathbf{T}_{\text {início decomposicão }}\left({ }^{\circ} \mathbf{C}\right)$ & Resíduo (\%) \\
\hline HDPE & 458,2 & --- \\
LLDPE & 454,2 & --- \\
HDPE/LLDPE & 456,0 & --- \\
NSC(2,5) & 449,7 & 2,5 \\
NSC(5,0) & 456,8 & 5,2 \\
NSC(7,5) & 449,8 & 7,0 \\
NC(2,5) & 457,4 & 2,6 \\
NC(5,0) & 454,8 & 5,2 \\
NC(7,5) & 452,5 & 6,2 \\
\hline
\end{tabular}

A Figura 4 apresenta os termogramas de DSC do HDPE, do LLDPE e da blenda (75/25\%). A influência da adição de argila organofílica no comportamento térmico é mostrada na Figura 5 e a influência da adição de agente

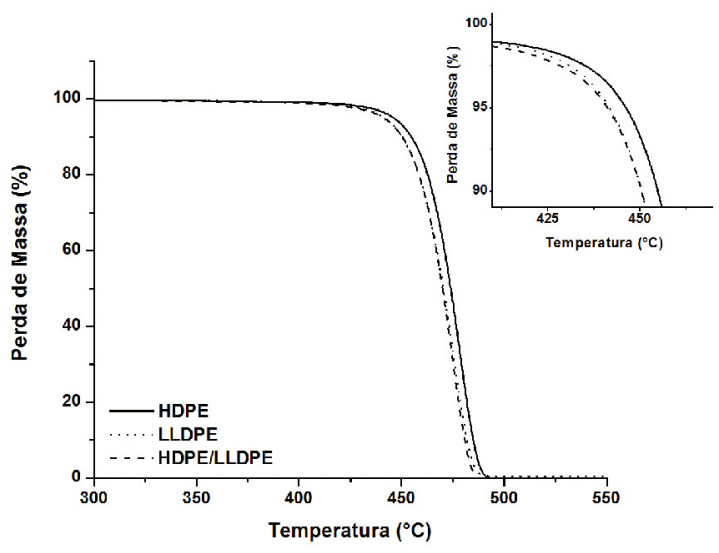

(a)

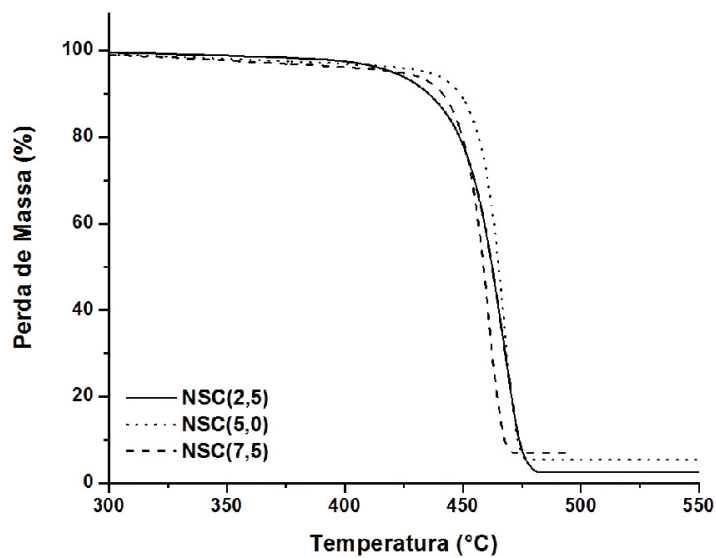

(b)

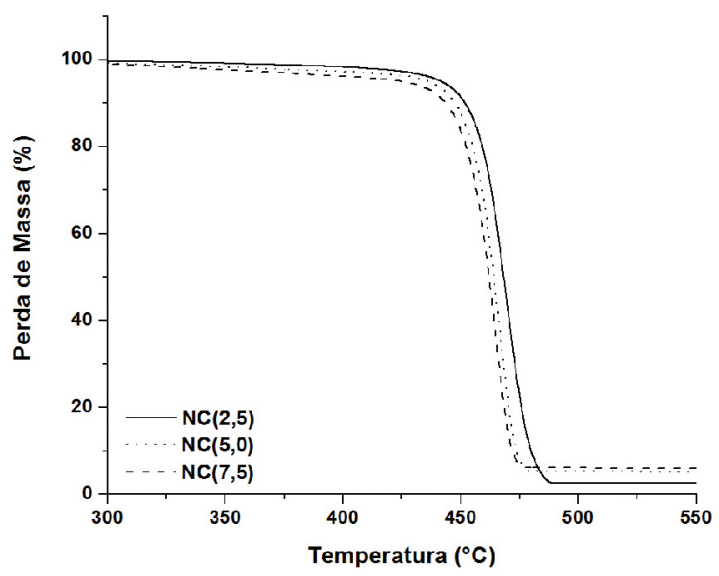

(c)

Figura 3. Curvas de TGA: (a) HDPE, LLDPE e blenda HDPE/ LLDPE (75/25\%), (b) Nanocompósitos HDPE/LLDPE/ OMMT sem adição de agente compatibilizante (NSC) e (c) Nanocompósitos HDPE/LLDPE/OMMT compatibilizados com HDPE-g-MA (NC). 


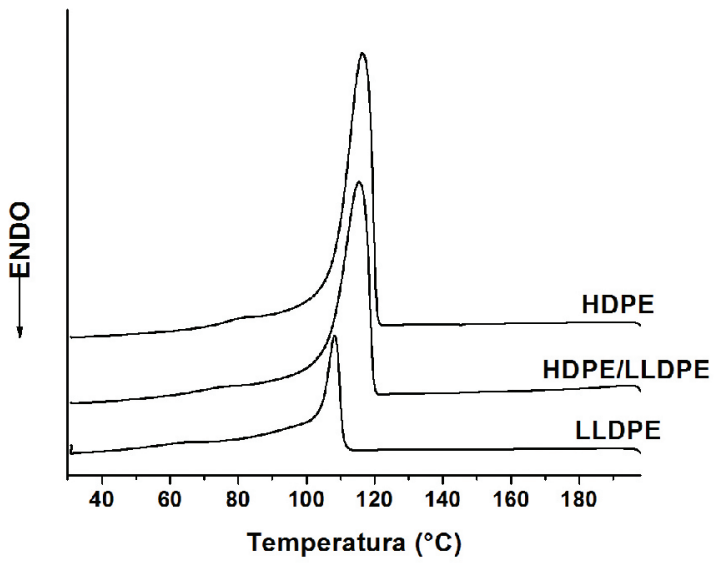

(a)

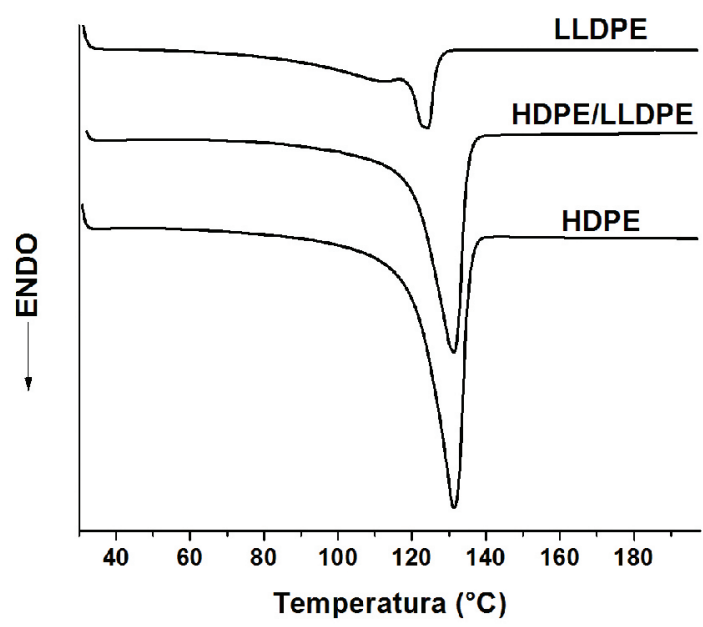

(b)

Figura 4. Termogramas de DSC do HDPE, LLDPE e da blenda HDPE/LLDPE (75/25\%): (a) resfriamento e (b) segundo ciclo de aquecimento.

compatibilizante no nanocompósito de HDPE/LLDPE/ OMMT é mostrada na Figura 6.

Os valores da temperatura de cristalização ( $\mathrm{T}$ ), temperatura de fusão $\left(\mathrm{T}_{\mathrm{m}}\right)$ e o grau de cristalinidade $\left(\mathrm{X}_{\mathrm{c}}\right)$ são mostrados na Tabela 2 .

Os valores de grau de cristalinidade dos nanocompósitos foram calculados considerando-se o teor de nanoargila, obtido por TGA, em cada sistema, ou seja, foi normalizado pelo teor de matriz polimérica em cada sistema. Os nanocompósitos, assim como a blenda polimérica, apresentaram uma única $\mathrm{T}_{\mathrm{m}}$ e $\mathrm{T}_{\mathrm{c}}$, indicando possível miscibilidade dos componentes no estado fundido e presença de co-cristalização, que pode ter possibilitado um aumento na nucleação, aumento da regularidade das cadeias poliméricas e formação de uma estrutura mais homogênea. Pode-se observar que a adição de nanoargila na blenda HDPE/LLDPE dificulta a nucleação diminuindo o grau de cristalinidade dos nanocompósitos (65\% para a blenda e $62 \%$ para nanocompósito com $2,5 \%$ de argila organofílica - NSC $(2,5))$. Por outro lado, o aumento da quantidade de nanoargila levou a um aumento no grau de cristalinidade desses nanocompósitos.

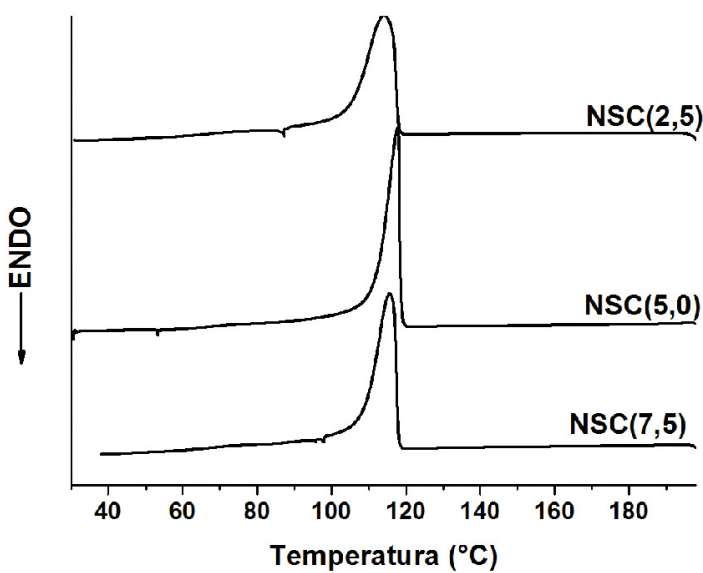

(a)

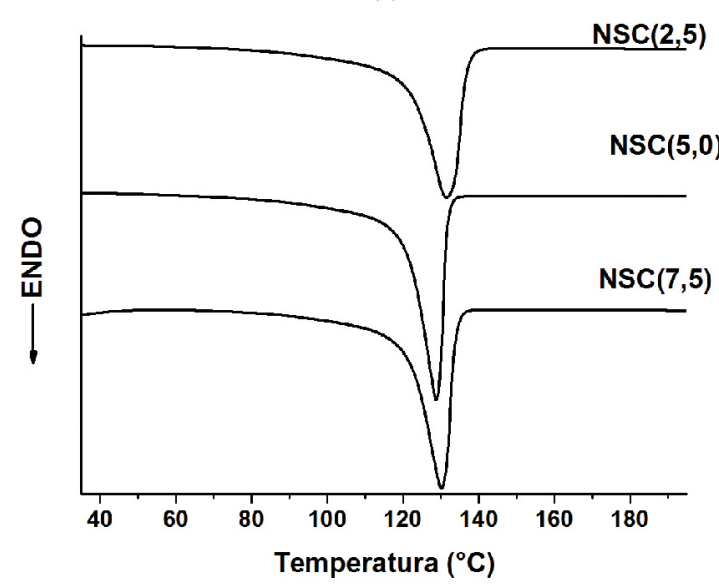

(b)

Figura 5. Termogramas de DSC dos nanocompósitos de blenda HDPE/LLDPE sem agente compatibilizante com diferentes teores de OMMT: (a) resfriamento e (b) segundo ciclo de aquecimento.

Neste caso, a argila organofílica atuou como um agente nucleante, aumentando a temperatura de cristalização (em relação a blenda HDPE/LLDPE) e reduzindo o tamanho do cristal ${ }^{[22]}$. Por outro lado, a adição do agente compatibilizante e o aumento no teor de nanoargila diminuem o grau de cristalinidade dos nanocompósitos. A maior interação do agente compatibilizante com a superfície do argilomineral gerou uma maior dificuldade para a nucleação e/ou crescimento dos cristais da matriz polimérica de HDPE/LLDPE, uma vez que há um comprometimento na mobilidade do sistema.

\section{Avaliação das propriedades de transporte}

O coeficiente de permeabilidade $(P)$ de um gás através de um polímero pode ser descrito em função dos coeficientes de solubilidade $(S)$ e difusão $(D)$ através da equação $P=D . S$, e existem alguns fatores que influenciam esses processos. A solubilidade é afetada com a facilidade de condensação do gás no interior do polímero, a presença de volume livre e a polaridade relativa do conjunto gás/matriz polimérica/carga ${ }^{[4,7,23]}$. $\mathrm{O}$ processo 
de difusão, por sua vez, é afetado também pela presença de volumes livres, pelo grau de cristalinidade da matriz polimérica, uma vez que a difusão ocorre exclusivamente na fase amorfa do polímero, e pela tortuosidade na matriz polimérica que aumenta o caminho livre médio para a difusão $0^{[4,7,23]}$.

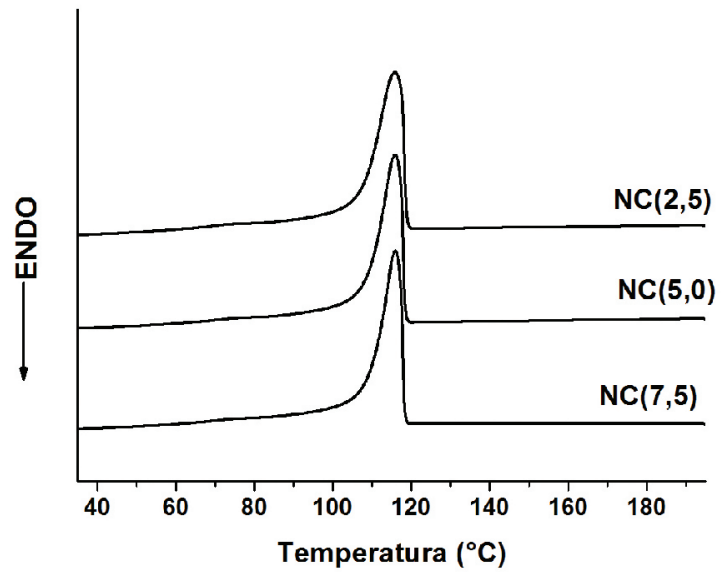

(a)

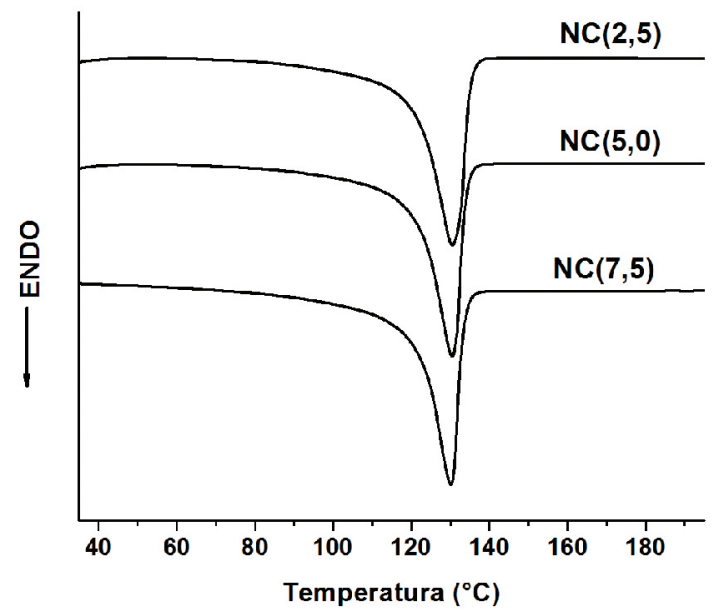

(b)

Figura 6. Termogramas de DSC dos nanocompósitos de blenda HDPE/LLDPE compatibilizados com HDPE-g-MA: (a) resfriamento e (b) segundo ciclo de aquecimento.
A Tabela 3 apresenta os valores dos coeficientes de permeabilidade a vapor de água, oxigênio e dióxido de carbono dos materiais estudados.

As poliolefinas, devido a sua natureza apolarhidrofóbica, são naturalmente excelentes barreiras ao vapor de água. O LLDPE possui menor grau de cristalinidade que o HDPE, ou seja, possui uma maior quantidade de fase amorfa que adsorve e permite a difusão de gases e vapores e dessa forma aumenta o coeficiente de permeabilidade quando comparado ao HDPE com maior grau de cristalinidade. No caso da blenda HDPE/LLDPE, a adição de $25 \%$ em massa de LLDPE levou à diminuição do coeficiente de permeabilidade ao vapor de água, sendo obtido valor menor até mesmo que o do HDPE puro, principalmente devido ao aumento do grau de cristalinidade observado decorrente da co-cristalização, havendo dessa forma menor difusão de vapor de água na blenda.

Analisando o comportamento para dióxido de carbono e oxigênio se espera que os coeficientes de permeabilidade sejam maiores que os valores obtidos para vapor de água, devido as suas naturezas apolares, o que de fato foi observado. No caso dos coeficientes de permeabilidade para o $\mathrm{CO}_{2}$ e $\mathrm{O}_{2}$, devem-se considerar as diferenças no tamanho e na condensabilidade destes gases. O coeficiente de sorção está relacionado com a energia requerida para o permeante ser sorvido pelo polímero e aumenta com um acréscimo da condensabilidade do permeante. Esta dependência significa que o coeficiente de sorção aumenta com o diâmetro molecular, já que moléculas maiores são normalmente mais condensáveis que as pequenas ${ }^{[24,25]}$, aumentando, neste caso, o coeficiente de permeabilidade do $\mathrm{CO}_{2}$.

Comportamento similar à blenda polimérica foi observado para os nanocompósitos de blenda HDPE/ LLDPE sem adição de agente compatibilizante, sendo barreira para o vapor de água e baixa barreira para $\mathrm{O}_{2}$ e $\mathrm{CO}_{2}$. Espera-se que a adição de cargas inorgânicas aumente o caminho livre médio para a difusão e que ocorra diminuição do coeficiente de permeabilidade ${ }^{[4,7]}$. Com a adição de nanoargila nas formulações dos nanocompósitos não foram observadas mudanças significativas no coeficiente de permeabilidade ao vapor de água. Nesses sistemas há a presença de aglomerados de argila e má dispersão da carga inorgânica na matriz, que podem auxiliar no aumento da quantidade de microvazios na matriz polimérica, facilitando a difusão e aumentando,

Tabela 2. Valores de temperatura de cristalização $\left(\mathrm{T}_{c}\right)$, temperatura de fusão $\left(\mathrm{T}_{\mathrm{m}}\right)$, entalpia de fusão $\left(\Delta \mathrm{H}_{\mathrm{m}}\right)$, grau de cristalinidade $\left(\mathrm{X}_{\mathrm{c}}\right)$ do HDPE, LLDPE, HDPE/LLDPE e nanocompósitos sem adição de agente compatibilizante.

\begin{tabular}{lcccc}
\hline \multicolumn{1}{c}{ Amostra } & $\mathbf{T}_{\mathbf{c}}\left({ }^{\circ} \mathbf{C}\right)$ & $\mathbf{T}_{\mathbf{m}}\left({ }^{\circ} \mathbf{C}\right)$ & $\Delta \mathbf{H m}(\mathbf{J} / \mathbf{g})$ & $\mathbf{X c}(\boldsymbol{\%})$ \\
\hline HDPE & 116,3 & 132,0 & 180,1 & 61,6 \\
LLDPE & 105,8 & 123,7 & 58,7 & 41,7 \\
HDPE/LLDPE & 112,8 & 133,8 & 164,7 & 64,7 \\
NSC(2,5) & 114,2 & 131,4 & 158,6 & 61,8 \\
NSC(5,0) & 116,7 & 131,1 & 157,7 & 63,0 \\
NSC(7,5) & 115,6 & 130,2 & 159,3 & 65,7 \\
NC(2,5) & 115,8 & 130,5 & 173,1 & 65,7 \\
NC(5,0) & 115,9 & 130,5 & 167,1 & 61,3 \\
NC(7,5) & 116,0 & 130,2 & 159,1 & 57,0 \\
\hline
\end{tabular}


Tabela 3. Valores do coeficiente de permeabilidade a vapor de água $\left(\mathrm{P}_{\mathrm{H}_{2} \mathrm{O}}\right)$, oxigênio $\left(\mathrm{P}_{\mathrm{O}_{2}}\right)$ e dióxido de carbono $\left(\mathrm{P}_{\mathrm{CO}_{2}}\right)$ dos materiais estudados.

\begin{tabular}{lccc}
\hline \multicolumn{1}{c}{ Amostra } & $\mathbf{P}_{\mathrm{H}_{2} \mathbf{O}}\left(\mathbf{1 0}{ }^{-5}\right.$ Barrer $)$ & $\mathbf{P}_{\mathbf{O}_{2}}($ Barrer $)$ & $\mathbf{P}_{\mathbf{C O}_{2}}(\mathbf{B a r r e r})$ \\
\hline HDPE & $5,9 \pm 0,8$ & $1,15 \pm 0,01$ & $5,88 \pm 0,79$ \\
LLDPE & $23,9 \pm 7,0$ & $4,11 \pm 0,01$ & $13,44 \pm 0,47$ \\
HDPE/LLDPE & $4,5 \pm 1,4$ & $1,44 \pm 0,22$ & $4,60 \pm 0,03$ \\
NSC(2,5) & $9,2 \pm 0,4$ & $2,28 \pm 0,05$ & $5,91 \pm 0,43$ \\
$\mathbf{N S C}(\mathbf{5 , 0})$ & $13,6 \pm 2,9$ & $1,83 \pm 0,41$ & $6,06 \pm 0,14$ \\
$\mathbf{N S C}(\mathbf{7 , 5})$ & $13,0 \pm 1,0$ & $0,98 \pm 0,05$ & $6,99 \pm 0,16$ \\
$\mathbf{N C}(\mathbf{2 , 5})$ & $8,3 \pm 0,6$ & $1,18 \pm 0,17$ & $5,19 \pm 0,42$ \\
$\mathbf{N C}(\mathbf{5 , 0})$ & $9,0 \pm 1,1$ & $1,16 \pm 0,36$ & $4,98 \pm 0,45$ \\
$\mathbf{N C}(\mathbf{7 , 5})$ & $11,3 \pm 0,1$ & $0,98 \pm 0,05$ & $4,15 \pm 0,32$ \\
\hline
\end{tabular}

dessa forma, o coeficiente de permeabilidade em relação à blenda polimérica. No caso dos gases $\mathrm{O}_{2}$ e $\mathrm{CO}_{2}$ observaram-se dois comportamentos distintos: quanto maior era o teor da nanocarga utilizado, maior era a permeabilidade do sistema ao dióxido de carbono, porém, por outro lado, menor para o oxigênio. Nesses nanocompósitos ocorre aumento do grau de cristalinidade com o aumento do teor de OMMT nos sistemas, ou seja, além da tortuosidade gerada pelos tactóides de argila, há uma menor fração de fase amorfa que contribui para o aumento do caminho difusional, diminuindo o coeficiente de permeabilidade efetivo, como observado para o $\mathrm{O}_{2}$.

A adição de altos teores de agentes compatibilizantes no nanocompósito pode ter alguns efeitos como diminuição da densidade dos domínios cristalinos na blenda, causar aumento do caráter amorfo e flutuações no volume livre ${ }^{[26]}$. Por outro lado, auxilia no processo de quebra de tactóides e dispersão de lamelas de argila na matriz polimérica auxiliando no aumento do caminho difusional do gás. Os resultados dos nanocompósitos compatibilizados com HDPE-g-MA não indicaram benefícios na adição da carga inorgânica quanto a barreira ao vapor de água, uma vez que quanto maior o teor da nanoargila, maior o teor adicionado do agente compatibilizante pois a relação argila: compatibilizante foi mantida; e este, devido a sua natureza química e menor permeabilidade intrínseca gera maior polaridade na matriz polimérica. Sendo a água uma substância também polar, maior torna-se o coeficiente de solubilidade e consequentemente, de permeabilidade deste permeante no polímero. Analisando as moléculas apolares, observou-se diminuição dos coeficientes de permeabilidade com o aumento do teor de OMMT. Comparando os resultados com a blenda polimérica nota-se diminuição da permeabilidade ao oxigênio para todas as composições estudadas. Para os teores de $2,5 \%$ e $5,0 \%$ em massa do argilomineral observa-se que os nanocompósitos compatibilizados com HDPE-gMA apresentaram permeabilidade a $\mathrm{CO}_{2}$ e $\mathrm{O}_{2}$ menores comparados aos nanocompósitos sem adição de agente compatibilizante, devido ao aumento da tortuosidade causado pelo estado intercalado e bem disperso das lamelas de argila na matriz polimérica observado nesses nanocompósitos, muito embora o HDPE-g-MA apresente intrinsicamente propriedades de barreira inferiores. A diminuição do coeficiente de permeabilidade está ligada a uma boa dispersão da carga inorgânica, boa molhabilidade desta pela matriz e forte interações na interface que diminuíram o número de microvazios, que poderiam ser facilitadores do processo difusional.

Baseando-se nos modelos de permeabilidade propostos por Nielsen ${ }^{[15]}$ e por Bharadwaj ${ }^{[16]}$, foi estimada a razão de aspecto $\mathrm{L} / \mathrm{W}$ das partículas de argilomineral das composições estudadas. Para tanto, foram obtidos gráficos de permeabilidade relativa $\left(\mathrm{P}_{\text {Nanocompósito }} / \mathrm{P}_{\text {Blenda }}\right.$ HDPE/LLDPE ) em relação a fração volumétrica de argila organofílica utilizada em cada composição. As simulações foram realizadas para o oxigênio como gás permeante, e são mostrados nas Figuras 7 e 8.

Pode-se observar nos gráficos que as composições N1(2,5), N1(5,0) (Figura 7) apresentaram valor de permeabilidade ao oxigênio superior a blenda polimérica, não se ajustando aos valores previstos pelos modelos de permeabilidade estudados. Os valores de L/W obtidos para cada modelo de permeabilidade e os valores de L/W calculados utilizando-se as micrografias obtidas por MET e o analisador de imagens Image-Pro Plus ${ }^{\circledR}$ são apresentados na Tabela 4. Para a obtenção do valor real de L/W foram analisadas 5 micrografias de diferentes regiões da amostra e o resultado obtido corresponde a média de 300 lamelas de OMMT analisadas para cada composição.

O processo de obtenção de filmes planos pode orientar as lamelas de argila na direção do estiramento na produção dos filmes. Porém, as condições de processamento utilizadas (velocidade de estiramento e vazão) e as condições de resfriamento do equipamento (ar forçado na saída da matriz plana e rolos com resfriamento controlado) utilizadas na preparação dos filmes planos dos nanocompósitos não foram suficientes para orientar e alinhar todas as lamelas de argila, onde se obteria o fator de orientação $S=1$, correspondente ao modelo de Nielsen, havendo certa quantidade de argila distribuída randomicamente na matriz (caso a distribuição fosse totalmente randômica, $S=0$ ). Assim, quanto ao fator orientação pode-se concluir que os nanocompósitos são constituídos em grande parte por lamelas orientadas em direção ao estiramento e com algumas lamelas distribuídas randomicamente na matriz polimérica, ou seja, com $\mathrm{S}$ variando entre 0 e 1 , porém mais próximo a 1 . Dessa forma, o modelo proposto por Nielsen e o modelo proposto por Bharadwaj com $\mathrm{S}=0$ apresentaram boa concordância com os valores medidos 


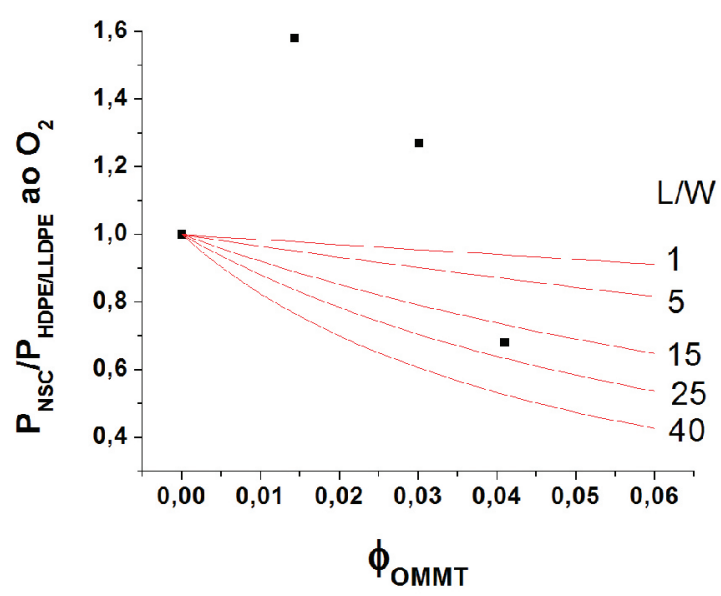

(a)

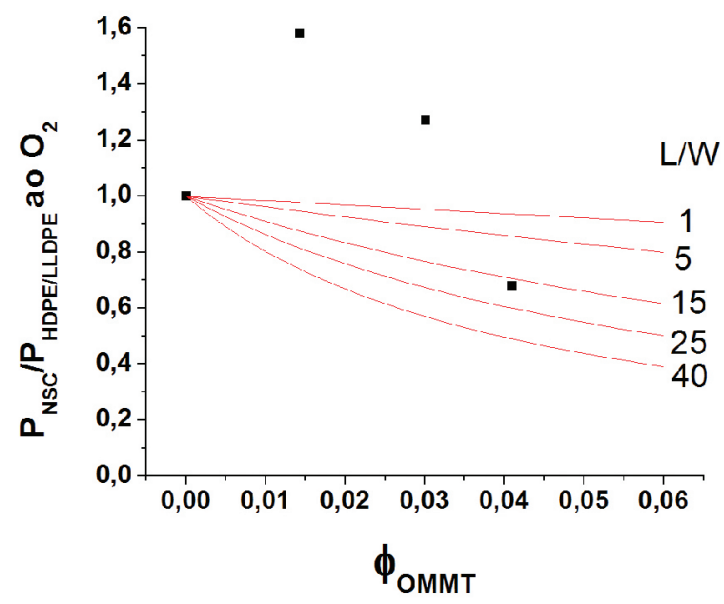

(b)

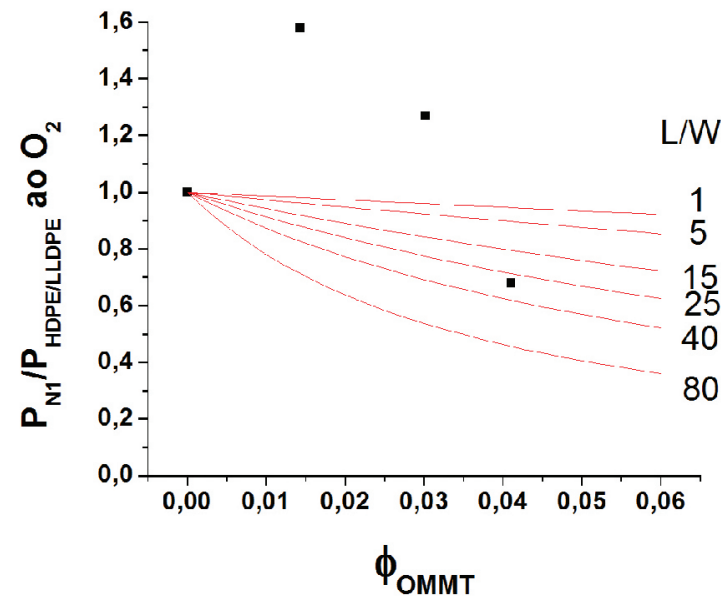

(c)

Figura 7. Curva da permeabilidade relativa dos nanocompósitos de blenda HDPE/LLDPE sem adição de agente compatibilizante em função da fração volumétrica de nanoargila: (a) Modelo de Nielsen, (b) Modelo de Bharadwaj; $\mathrm{S}=0$ e (c) Modelo de Bharadwaj; $\mathrm{S}=-1 / 2$.

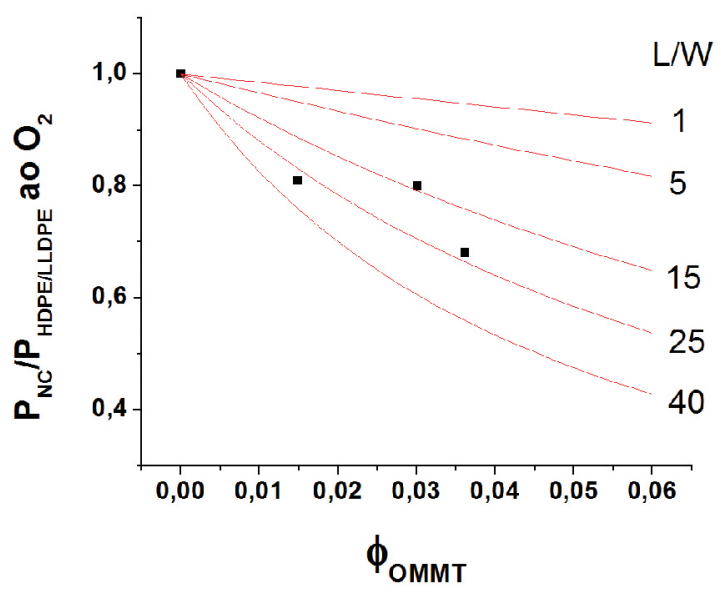

(a)

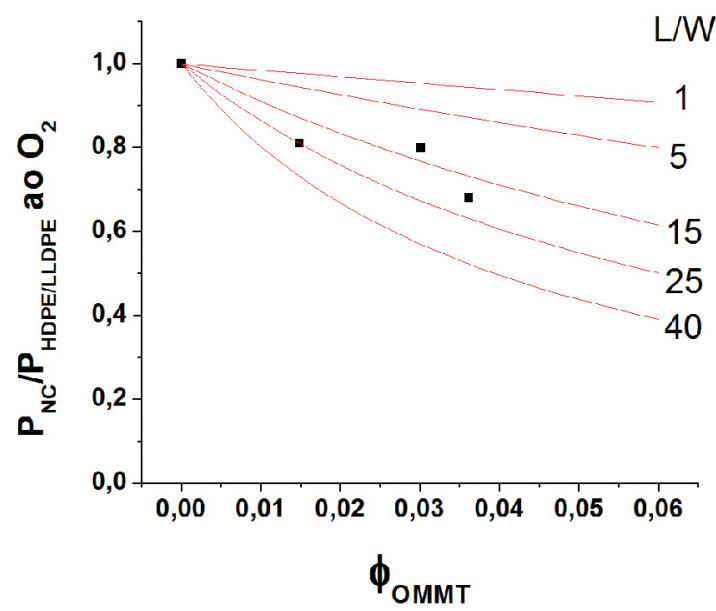

(b)

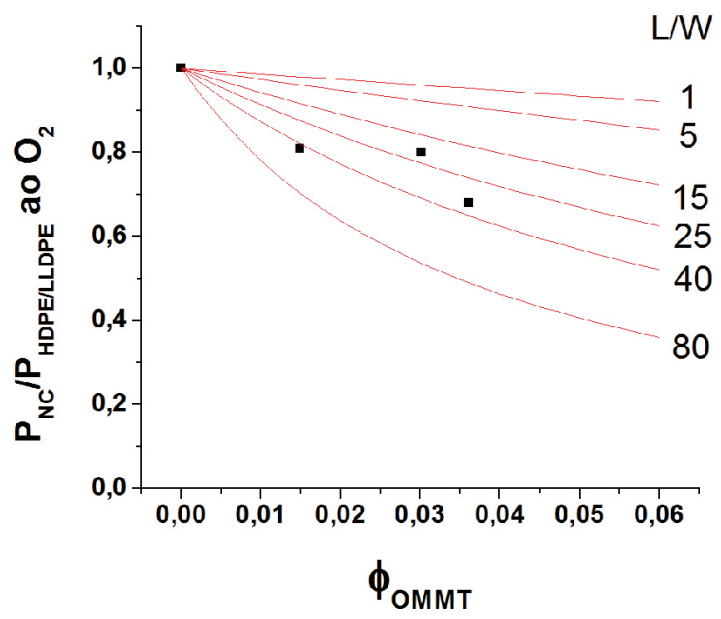

(c)

Figura 8. Curva da permeabilidade relativa dos nanocompósitos de blenda HDPE/LLDPE com HDPE-g-MA em função da fração volumétrica de nanoargila: (a) Modelo de Nielsen, (b) Modelo de Bharadwaj; $\mathrm{S}=0$ e (c) Modelo de Bharadwaj; $\mathrm{S}=-1 / 2$. 
Tabela 4. Valores de L/W das amostras estudadas obtidas através de diferentes modelos de permeabilidade.

\begin{tabular}{lcccc}
\multicolumn{1}{c}{ Amostra } & $\begin{array}{c}\text { L/W } \\
\text { Nielsen }\end{array}$ & $\begin{array}{c}\text { L/W } \\
\text { Bharadwaj } \\
\text { S=0 }\end{array}$ & $\begin{array}{c}\text { L/W } \\
\text { Bharadwaj } \\
\text { S=-1/2 }\end{array}$ & $\begin{array}{c}\text { L/W } \\
\text { MET* }\end{array}$ \\
\hline NSC(2,5) & --- & -- & --- & $3,2 \pm 0,9$ \\
NSC(5,0) & --- & -- & -- & $3,7 \pm 1,1$ \\
NSC(7,5) & 20 & 17 & 27 & $5,7 \pm 2,7$ \\
NC(2,5) & 30 & 25 & 42 & $18,7 \pm 4,8$ \\
NC(5,0) & 15 & 13 & 20 & $10,7 \pm 3,0$ \\
NC(7,5) & 22 & 19 & 35 & $15,5 \pm 5,1$ \\
\hline
\end{tabular}

*Valores médios obtidos através das micrografias de MET e utilização do analisador de imagens Image-Pro Plus®.

Tabela 5. Propriedades óticas das composições estudadas.

\begin{tabular}{lccc}
\hline \multicolumn{1}{c}{ Composição } & Transmitância (\%) & Opacidade (\%) & Claridade (\%) \\
\hline HDPE & $88,4 \pm 1,5$ & $40,2 \pm 1,7$ & $44,3 \pm 1,8$ \\
LLDPE & $89,0 \pm 1,1$ & $47,8 \pm 2,4$ & $74,8 \pm 2,4$ \\
HDPE/LLDPE & $89,1 \pm 0,7$ & $27,7 \pm 1,8$ & $58,1 \pm 3,3$ \\
NSC (2,5) & $89,5 \pm 1,0$ & $43,9 \pm 2,2$ & $36,5 \pm 2,2$ \\
NSC(5,0) & $87,3 \pm 1,5$ & $69,7 \pm 1,7$ & $15,7 \pm 0,6$ \\
NSC(7,5) & $85,1 \pm 2,9$ & $81,8 \pm 1,0$ & $9,82 \pm 0,3$ \\
NC(2,5) & $90,6 \pm 1,2$ & $30,0 \pm 4,2$ & $61,3 \pm 4,2$ \\
NC(5,0) & $89,3 \pm 1,3$ & $48,5 \pm 3,4$ & $39,8 \pm 1,1$ \\
NC(7,5) & $88,3 \pm 1,2$ & $69,5 \pm 1,7$ & $21,0 \pm 0,9$ \\
\hline
\end{tabular}

através da micrografia dos nanocompósitos. Já o modelo de Bharadwaj para $S=-1 / 2$ superestimou o valor de $\mathrm{L} / \mathrm{W}$ das composições.

\section{Avaliação das propriedades óticas}

Filmes de poliolefinas são muito utilizados no setor de embalagem, onde transparência e claridade são usualmente exigências básicas para esta aplicação. A opacidade também é uma propriedade muito importante, uma vez que filmes com baixa opacidade são mais transparentes.

As propriedades óticas dos materiais estudados foram analisadas através da medida de opacidade, claridade e transmitância, apresentadas na Tabela 5. A opacidade é a porcentagem de luz transmitida que, passando através da amostra, desvia do raio incidente pelo espalhamento da mesma em um ângulo maior que $2,5^{\circ}$, e depende apenas da superfície do filme. A claridade é a porcentagem de luz transmitida que, passando através da amostra, desvia do raio incidente pelo espalhamento da mesma em um ângulo menor que $2,5^{\circ}$. A transmitância é o total da luz incidente que é transmitida, e é reduzida pela reflectância e pela absorção.

Quanto maior o grau de cristalinidade maior deve ser a opacidade do material polimérico, porém a opacidade da blenda HDPE/LLDPE, com maior grau de cristalinidade, diminui em relação aos materiais puros (HDPE e LLDPE), nesse caso a co-cristalização possibilitou a formação de cristais com menor tamanho aparente, podendo ser menor que o comprimento de onda da luz visível, fazendo com o espalhamento seja consideravelmente menor do que para o HDPE e LLDPE, resultando em maior transparência.

A adição de argila organofílica na blenda polimérica aumentou a opacidade, no entanto, a adição do agente compatibilizante diminuiu a opacidade comparativamente aos nanocompósitos sem adição de agente compatibilizante, como pode ser observado para a composição NSC(2,5) com 43,9\% de opacidade e com a adição de HDPE-g-MA como agente compatibilizante observou-se uma diminuição da opacidade para 30,0\% da composição $\mathrm{NC}(2,5)$.

De maneira geral, observou aumento da opacidade, diminuição da transmitância e claridade com o aumento do teor de argila nos nanocompósitos. Por fim, os filmes dos nanocompósitos compatibilizados com adição de 2,5\% de OMMT apresentaram opacidade menor que os materiais puros (HDPE e LLDPE), podendo ser utilizados no setor de embalagem.

\section{Conclusões}

A avaliação térmica dos nanocompósitos de blenda HDPE/LLDPE e OMMT mostrou que a argila organofílica atuou como agente nucleante, aumentando o grau de cristalinidade com o aumento do teor na composição. Por outro lado, a adição de agentes compatibilizantes dificultou a nucleação e/ou crescimento dos cristais, diminuindo o grau de cristalinidade com o aumento do teor de argilomineral

A avaliação das propriedades de transporte mostrou que a blenda HDPE/LLDPE e os nanocompósitos comportam-se como barreira ao vapor de água e são permeáveis ao $\mathrm{CO}_{2}$ e $\mathrm{O}_{2}$. A adição de nanoargila modificou o grau de cristalinidade dos nanocompósitos e a diminuição do coeficiente de permeabilidade foi decorrência do aumento do grau de cristalinidade e do aumento do caminho difusional do gás, pelo aumento da tortuosidade. A diminuição do coeficiente de permeabilidade está relacionada a uma boa dispersão da 
carga inorgânica, boa molhabilidade desta pela matriz e forte interações na interface que diminuíram o número de microvazios, que poderiam ser facilitadores do processo difusional. Os modelos teóricos de permeabilidade proposto por Nielsen e Bharadwaj são boas ferramentas para estimar a razão de aspecto da nanoargila nos nanocompósitos.

Quanto as propriedades óticas, observou-se aumento da opacidade, diminuição da transmitância e claridade com o aumento do teor de argila nos nanocompósitos

\section{Agradecimentos}

Os autores agradecem ao CNPq e a FAPESP pelo apoio financeiro.

\section{Referências Bibliográficas}

1. Alexandre, M. \& Dubois, P. - Mat. Sci. Eng., 28, p.1 (2000). http://dx.doi.org/10.1016/S0927-796X(00)00012-7

2. Lomakim, S. M.; Novokshonova, L. A.; Brevnov, P. N. \& Shchegolikhin, A. N. - J. Mat. Sci., 43, p.1340 (2008). http://dx.doi.org/10.1007/s10853-007-2295-1

3. Luo, J. J. \& Daniel, I. M. - Comp. Sci. Tech., 63, p.1607 (2003). http://dx.doi.org/10.1016/S0266-3538(03)00060-5

4. Picard, E.; Vermogen, A. Gérard, J-F. \& Espuche, E. - J. Polym. Sci. B Polym. Phis., 46, p.2593 (2008).

5. Spencer, M. W.; Cui, L.; Yoo, Y. \& Paul, D. R. - Polymer, 51, p.1056 (2010). http://dx.doi.org/10.1016/j. polymer.2009.12.047

6. Ryu, S. H. \& Chang, Y. W. - Polym. Bull., 55, p.385 (2005). http://dx.doi.org/10.1007/s00289-005-0437-7

7. Durmus, A.; Woo, M.; Kasgoz, A.; Macosko, C. W. \& Tsapatsis, M. - Eur. Polym. J., 43, p.3737 (2007).

8. Hotta, S. \& Paul, D. R. - Polymer, 45, p.7639 (2004). http:// dx.doi.org/10.1016/j.polymer.2004.08.059

9. Gopakumar, T. G.; Lee, J. A.; Kontopoulou, M. \& Parent, J, S. - Polymer, 43, p.5483 (2002). http://dx.doi.org/10.1016/ S0032-3861(02)00403-2

10. Wang, K. H.; Choi, M. H.; Koo, C. M.; Choi, Y. S. \& Chung, I. J. - Polymer, 42, p.9819 (2001). http://dx.doi. org/10.1016/S0032-3861(01)00509-2

11. Comyn, J. - "Polymer permeability", Elsevier Applied Science Publishers, London (1998).

12. Lee, J. H.; Jung, D.; Hong, C. E.; Rhee, K. Y. \& Advani, S. G. - Comp. Sci. Tech., 65, p.1996 (2005). http://dx.doi. org/10.1016/j.compscitech.2005.03.015
13. Kato, M.; Okamoto, N.; Hasegawa, A.; Tsukigase, A. \& Usuki, A. - Polym. Eng. Sci., 43, p.1312 (2003). http:// dx.doi.org/10.1002/pen.10111

14. Morales, A. R.; Cruz, C.; Peres, L. \& Ito, E. N. - Polímeros, 20, p.39 (2010). http://dx.doi.org/10.1590/ S0104-14282010005000004

15. Nielsen, L. E - J. Macromol. Sci., 5, p.929 (1967). http:// dx.doi.org/10.1080/10601326708053745

16. Bharadwaj, R. K. - Macromolecules, 34, p.9189 (2001). http://dx.doi.org/10.1021/ma010780b

17. Passador, F.R.; Backes, E. H.; Travain, D. R.; Ruvolo Filho, A. C. \& Pessan, L. A. - Polímeros, 23, p.521 (2013). http:// dx.doi.org/10.4322/polimeros.2013.056

18. American Society for Testing and Materials - ASTM - "ASTM E 96/E96M-05: Standard test methods for water vapor transmission of materials", ASTM (2005).

19. American Society for Testing and Materials - ASTM - "ASTM D3985-05: Standard test methods for oxygen gas transmission rate through plastic film and sheeting using a coulometric sensor", ASTM (2005).

20. American Society for Testing and Materials - ASTM - "ASTM D2476-05: Standard test methods for the determination of carbon dioxide gas transmission rate (CO2TR) through barrier materials using an infrared detector", ASTM (2005).

21. American Society for Testing and Materials - ASTM - "ASTM D1003-07: Standard test methods for haze and luminous transmittance of transparent plastics", ASTM (2007)

22. Min, K. D.; Kim, M. Y.; Choi. K. Y.; Lee, J. H. \& Lee, S. G. - Polym. Bull., 57, p.101 (2006). http://dx.doi. org/10.1007/s00289-006-0537-z

23. Koros, W. J.; Moaddeb, M. "Gas barrier polymer", in: Polymeric materials encyclopedia, J. C. Salamone (ed.), CRC Press, New York (1996).

24. Baker, R. W. - "Membrane Technology and Applications", John Wiley \& Sons, Chichester (2004). http://dx.doi. org/10.1002/0470020393

25. Stern, S. A. - J. Membrane Sci., 94, p.1 (1994). http:// dx.doi.org/10.1016/0376-7388(94)00141-3

26. Gugliuzza, A. \& Drioli, E. - Polymer, 46, p.9994 (2005). http://dx.doi.org/10.1016/j.polymer.2005.08.011

Enviado: $30 / 07 / 12$ Reenviado: $17 / 09 / 12$ Aceito: $22 / 10 / 12$ 